\title{
Lektüren des Ästhetischen - Ästhetische Lektüren. Alte und neue Hermeneutik
}

\author{
Annette Gerok-Reiter
}

Eingegangen: 21. September 2021 / Angenommen: 30. September 2021 / Online publiziert: 1. Dezember 2021

(C) Der/die Autor(en) 2021

Zusammenfassung Lektüren des Ästhetischen bilden einen >Paradefall $<$ hermeneutischen Verstehens. Zugleich jedoch kommt der hermeneutische Zugriff gerade hier an seine Grenze, werden Lektüren des Ästhetischen zu dessen >Härtefall<. Die grundsätzliche Skepsis gegenüber einem hermeneutischen Zugriff ebenso wie gegenüber Interpretationen, die vorrangig ästhetische Aspekte fokussieren, ist daher in den letzten Jahrzehnten durchaus in Relation zu sehen. Vor diesem Hintergrund möchte die Skizze pointieren, inwiefern die derzeitige >Rückkehr der Ästhetik $<$ mit einem Perspektivwechsel korreliert ist, der auch eine Neujustierung hermeneutischen Verstehens einfordert.

Schlüsselwörter Germanistik · Literaturwissenschaft · Ästhetik · Hermeneutik · Lesen $\cdot$ Verstehen · Interpretieren · Aesthetic turn · Andere Ästhetik 


\title{
Readings of the Aesthetic - Aesthetic Readings. Old and New Hermeneutics
}

\begin{abstract}
Readings of the aesthetic are a prime example of hermeneutic interpretation. By the same token however, these readings of the aesthetic are what puts the hermeneutic approach to the test and pushes it to its limit. For the last few decades, the general suspicions of hermeneutic approaches and of interpretations which mainly focus on aesthetic aspects should therefore be seen as interrelated. Against this backdrop, this outline wishes to draw special attention to the fact that the current >comeback of aesthetics< comes with a change of perspective which also calls for hermeneutic interpretation to be recalibrated.
\end{abstract}

Keywords German Studies · Literary Studies - Aesthetics · Hermeneutics · Reading · Understanding · Interpreting · Aesthetic Turn · Different Aesthetics

Lektüren des Ästhetischen, d. h. Lektüren ästhetisch modellierter Texte, ${ }^{1}$ bieten in der Regel einen >Paradefall < für den hermeneutischen Zugriff, insbesondere in der Perspektive der >älteren< Hermeneutik von Wilhelm Dilthey über Hans-Georg Gadamer bis hin zu Hans Robert Jauß. ${ }^{2}$ Die enge Korrelation von Hermeneutik und Ästhetik erscheint dabei naheliegend. So setzen Lektüren des Ästhetischen in hohem Maß die souveräne Kenntnis der jeweiligen Sprache und das Wissen um textkonstituierende Komponenten als Grundbedingungen eines gelingenden Leseprozesses voraus, d. h. die Regeln eines grammatischen, philologiebasierten Verstehens gemäß Schleiermacher. ${ }^{3}$ Ebenso entziehen sie sich einem oberflächlichen, rein konsumierenden Lesen, sondern drängen auf Reflexion und Interpretation, wollen sie in Prozessen der Sinnkonstitution über das Ideal des delectare hinauskommen. Und sie verweigern sich dezidiert einem Urteil nach den Oppositionskriterien von richtig oder falsch: Der Anspruch des Verstehens zielt auf komplexere Relationen. ${ }^{4}$ Hermeneutische Bedeutungserschließung gehört daher seit den Anfängen der Literaturwissenschaft zu den notwendigen methodischen Praktiken ästhetischer Lektüren.

\footnotetext{
1 Der Begriff der Lektüre wird hier als Terminus benutzt, der im umfassenden Sinn die Akte des Lesens, Interpretierens und Verstehens umfasst, also nicht zwingend eine Verkürzung bedeuten muss. Vgl. Lobsien, Eckhard: Schematisierte Ansichten. Literaturtheorie mit Husserl, Ingarden, Blumenberg. Paderborn/ München: Fink, 2012, S. $11 \mathrm{f}$.

2 Dilthey, Wilhelm: Das Erlebnis und die Dichtung. Lessing. Goethe, Novalis, Hölderlin (Gesammelte Schriften, Bd. 26). Göttingen: Vandenhoeck \& Ruprecht, 2005 [1906]; Gadamer, Hans-Georg: Hermeneutik I. Wahrheit und Methode. Grundzüge einer philosophischen Hermeneutik. Tübingen: Mohr, 4. Aufl. 1975, insbes. »Erster Teil: Freilegung der Wahrheitsfrage an der Erfahrung der Kunst «, S. 11-161; Jauß, Hans Robert: Ästhetische Erfahrung und literarische Hermeneutik. Bd. 1: Versuche im Feld der ästhetischen Erfahrung. München: Fink, 1977.

3 Vgl. Schleiermacher, Friedrich D. E.: Hermeneutik und Kritik. Hg. und eingel. von Manfred Frank. Frankfurt a. M.: Suhrkamp, 1977, S. $80 \mathrm{f}$.

4 Siehe zu den genannten Kriterien auch Bleumer, Hartmut u. a.: »Bindestrich-Hermeneutiken - Neue Verortungen der Lektüre?« In: Zeitschrift für Literaturwissenschaft und Linguistik 50 (2021), S. 563-580, hier S. 564f. Dass dieses komplexere Verstehen - gegen Schleiermacher - kein psychologisch-intuitives Verstehen bedeuten muss, ist durch die Vorgaben der Reflexion und Interpretation bereits impliziert.
} 
Zugleich jedoch wird die hermeneutische Bedeutungserschließung im Feld des Ästhetischen an ihre Grenze geführt, werden Lektüren des Ästhetischen zum >Härtefall< und zur Provokation hermeneutischen Verstehens. Der Grund hierfür lässt sich als Kehrseite dessen verstehen, was in den Lektüren des Ästhetischen die hermeneutische Erschließung an und für sich gerade einfordert - und dies in dreierlei Hinsicht: Erstens führt der ästhetische Anspruch des Textes selbst an jene Grenze, insofern er eben das als >ästhetisch < auszeichnet, was in keiner propositionalen Aussage aufgeht, ja, was sich dem Begriff grundsätzlich entzieht. Indem der ästhetische Anspruch auf das Unbestimmbare als das Ungreifbare abzielt, lässt er auch das Unsagbare, das gleichwohl in der Sprache aufgehoben ist, ja, lässt er Sprache überhaupt hinter sich. Ein hermeneutisches Verstehen, das sich auf das Erschließen primär sprachlich konstituierter Bedeutung ebenso wie sprachlich vermittelter Wahrheit von Texten ausrichtet, verfängt sich damit in Widersprüchen. ${ }^{5}$ Schwierigkeit bietet - zweitens - der ästhetische Gegenstand auch deshalb, als er in prononcierter Weise seine jeweilige Materialität und Medialität nicht nur in den Blick rückt, sondern ihm besonderes Gewicht zuspricht (hierzu zählen Rhythmus, Reim, Klang, Lautung, rhetorische Figuren, insbes. Strategien der Bildlichkeit, der synästhetischen Erfahrung; Fragen der Mündlichkeit, der Schriftlichkeit, der materialen Textkultur etc.). ${ }^{6}$ Ästhetisches Wahrnehmen, das auf dieses Angebot reagiert, ist daher von sinnlicher Affizienz nicht $\mathrm{zu}$ trennen. ${ }^{7} \mathrm{Ob}$ und wie von hier aus ein rationales Verstehen möglich ist oder - noch intrikater - wie sinnliche Erfahrung in Erkennen umschlagen kann, gehört zu den wohl meist reflektierten Irritationen philosophischer Ästhetik. Wenn Hermeneutik jedoch Wahrnehmen mit Verstehen und Erkennen verbindet, ${ }^{8}$ wird sie gerade hier auf eine besondere Probe gestellt. Und schließlich - drittens - bringen ästhetische Präsentationen in der Regel multiple Sinnebenen ins Spiel (von Phänomenen der Ambiguität über Angebote mehrfachen Schriftsinns bis hin zu nicht abschließbaren Lektürebewegungen der Dekonstruktion; in der Spannung von histoire und discours; im Integral von Sinnlichkeit und Sinn; in intermedialen Phänomenen etc.). Die Multiplikation der Sinnebenen droht den hermeneutischen Zugriff, sofern dieser sich in klassischer Weise Fragen »nach dem Autor, der Intention, der Tradition und der ontologischen Grundlage $\ll^{9}$ widmet, zu überfordern.

\footnotetext{
5 Vgl. zu der Problematik bei Gadamer, aber auch bei Dilthey Jung, Werner: »Neuere Hermeneutikkonzepte. Methodische Verfahren oder geniale Anschauung?« In: Klaus-Michael Bogdal (Hg.): Neue Literaturtheorien. Eine Einführung. Opladen: Westdt. Verl., 1997 [1990], S. 159-180, hier S. 165 und S. 171; grundsätzlich: Nassen, Ulrich: »Statt einer Einleitung: Notizen zur philologischen Hermeneutik «. In: Ders. (Hg.): Texthermeneutik. Aktualität, Geschichte, Kritik. Paderborn u. a.: Schöningh, 1979, S. 9-22, hier S. $10 \mathrm{f}$.

6 Zur Materialität von Texten: Seel, Martin: Ästhetik des Erscheinens. München: Hanser, 2000, S. 204-214.

7 Dazu insbes. Gumbrecht, Hans Ulrich: Diesseits der Hermeneutik. Die Produktion von Präsenz. Frankfurt a. M.: Suhrkamp, 2004, S. 17-37.

8 Vgl. Bleumer u. a. (s. Anm. 4), S. 568 f.

9 Vgl. ebd., S. 579. Zur Rehabilitierung eines relativierten Autorbegriffs und eine hypothetischen Intentionalismus im Sinn einer Neohermeneutik: Köppe, Tilmann/Winko, Simone: Neuere Literaturtheorien. Eine Einführung. 2., aktualisierte und erweiterte Aufl. Stuttgart/Weimar: J. B. Metzler, 2013, S. 133-148.
} 
Die Spannung zwischen Parade- und Härtefall ästhetischen Fragens im Rahmen der Hermeneutik prägt sich wissenschaftsgeschichtlich analog zur Spannung zwischen der Hermeneutik als methodischem Primat in den Geisteswissenschaften und einer grundlegenden Skepsis ihr gegenüber aus. So sind einerseits im Zuge der hermeneutischen Skepsis, andererseits durch die Überforderung des hermeneutischen Zugangs über mehrere Jahrzehnte genuin ästhetische Fragen im Wissenschaftsdiskurs vielfach in den Hintergrund getreten, verbunden mit der kulturwissenschaftlichen Wende der Geisteswissenschaften, die vom schwierigen Geschäft der Bedeutungs- und Sinnsuche aus dem Text heraus den Blick grundsätzlich auf Kontexte umlenken oder von posthermeneutischen Standpunkten aus die Bedeutungs- und Sinnsuche ganz in Frage stellen wollte: ${ }^{10} \gg$ Die neuere Hermeneutik gibt [...] die Vorstellung eines ursprünglichen, mit sich selbst identischen Textsinns auf, den es durch die Interpretation freizulegen gelte. ${ }^{11}$ Verzichtet die Literaturwissenschaft aber auf die hermeneutische Bedeutungs- und Sinnsuche, so verlieren nicht nur ästhetische Lektüren insgesamt an Glaubwürdigkeit und Geltung; die Literaturwissenschaft selbst droht, wie Klaus-Michael Bogdal zu Recht anmahnt, »belanglos«, weil beliebig zu werden. ${ }^{12}$ Von hier aus stellt sich die Frage, wie das hermeneutische Angebot der >Deutung < als literaturwissenschaftliche Kernaufgabe aufrecht erhalten bleiben kann, ohne dass die » Wahrheit< eines Textes $«{ }^{13}$ jenseits der ihm eigenen Geschichtlichkeit oder ein »mit sich selbst identische[r] Textsinn $\ll{ }^{14}$ jenseits ebenso dynamischer wie irritierender Fremdreferenzen gesucht werden müsste.

Es erscheint vor diesem Hintergrund plausibel, dass die $>$ Rückkehr der Ästhetik ${ }^{\wedge} 15$ in die geistes- und kulturwissenschaftlichen Diskussionen in zentraler Weise mit einer Neujustierung hermeneutischen Vorgehens in Verbindung steht. Denn die $>$ Rückkehr der Ästhetik< profitiert von einem entscheidenden methodischer Perspektivwechsel. Dessen Impuls verdankt sich über weite Strecken dem insbesondere in den Gesellschafts- und Naturwissenschaften in den letzten Jahren ausgerufenen aesthetic turn, ${ }^{16}$ ebenso aber auch den historisch arbeitenden Geisteswissenschaften, die sich dezidiert auf die Kulturwissenschaften hin geöffnet haben. Das vermeintliche

10 Vgl. Rusterholz, Peter: »Zum Verhältnis von Hermeneutik und neueren antihermeneutischen Strömungen«. In: Heinz Ludwig Arnold/Heinrich Detering (Hg.): Grundzüge der Literaturwissenschaft. München: dtv, 1996, S. 157-177.

11 Bogdal, Klaus-Michael: »Problematisierung der Hermeneutik im Zeichen des Poststrukturalismus«. In: Heinz Ludwig Arnold/Heinrich Detering (Hg.): Grundzüge der Literaturwissenschaft. München: dtv 1996, S. 137-156, hier S. 139-154, Zitat S. 147.

12 Ebd., S. 156.

13 Ebd., vgl. auch Nassen (s. Anm. 5), S. 20.

14 Bogdal (s. Anm. 11), S. 147.

15 Diese wird besonders deutlich in verschiedenen Forschungsprojekten, die sich in jüngerer Zeit wieder um dezidiert ästhetische Fragestellungen gruppieren: Siehe z. B. die DFG-Kolleg-Forschungsgruppe 1627 BildEvidenz. Geschichte und Ästhetik (FU Berlin), die SNF-Forschungsgruppe The Power of Wonder - The Instrumentalization of Admiration, Astonishment and Surprise in Discourses of Knowledge, Power and Art (Universität Zürich) oder den SFB 1391 Andere Ästhetik (Universität Tübingen).

16 In Auswahl: Opondo, Samson/Shapiro, Michael J. (Hg.): The New Violent Cartography. Geo-Analysis after the Aesthetic Turn. Abingdon, Oxon/New York: Routledge, 2012; Shapiro, Michael: Studies in transdisciplinary method. After the aesthetic turn. Abingdon (Oxon)/New York: Routledge, 2013; Kompridis, Nikolas: The Aesthetic Turn in Political Thought. New York/London: Bloomsbury Academic, 2014; Zeki, 
>Defizit< gerade vormoderner Artefakte, ihre fehlende Autonomie aufgrund ihrer lebensweltlichen Eingebundenheit, gewinnt in dieser Perspektive neue Attraktivität. In beiden Ansätzen tritt eine funktionale Ausrichtung von ästhetischen Gegenständen in konkreten lebensweltlichen, soziokulturellen Kontexten in den Vordergrund. Fremdreferenzen erhalten vor diesem Hintergrund eine veritable Geltung. Und statt die >Wahrheit< von Artefakten in einer der Zeit entzogenen Tiefendimensionen zu suchen, wird hier von den Auswirkungen oder der >sozialen Energie< im Rahmen konkreter gesellschaftlicher bzw. politischer Praktiken gesprochen; Artefakte werden in neuer Weise von heterologen Kontexten her begriffen. ${ }^{17}$ Im Gegensatz zu den 70er Jahren des letzten Jahrhunderts kommt dabei aber nun gerade den historisch arbeitenden Kulturwissenschaften die Aufgabe zu, einsinnig sozialhistorischkausale Programmatiken als Verkürzung, die auch in den derzeitigen Natur- und Gesellschaftswissenschaften droht, aufzudecken im Rekurs auf die Widerständigkeiten des ästhetischen Materials, seiner formalen Eigenlogik oder seiner pluralen Traditionsimplemente, wie umgekehrt die jeweils gestaltenden Elemente auf der autologischen Seite des Artefakts an die heterologischen Impulse gebunden bleiben, deren Diskursabhängigkeiten, Geschichtlichkeit und Unzuverlässigkeit mittransportieren, sich je neu im epistemischen Transfer anpassen müssen.

Damit greift eine doppelte Dynamisierung:18 ein >unstillbarer< Austausch an Wechselbeziehungen zwischen der autologischen Dimension der Texte einerseits, ihrer heterologischen Dimension andererseits - und dies nicht, indem ein Text >in einem geistesgeschichtlichen $>\mathrm{Um}<$-feld situiert würde, sondern indem sich dieser Austausch bis in kleinste Details der ästhetischen Faktur selbst hineinzieht, ebenso wie der Austausch von den sublimen Irritationen auf der Form-, Motiv- oder Gestaltebene immer erneut angetrieben wird. Dieser Dynamik kann jedoch nur eine $>$ neue $<$ und >andere $<$ Hermeneutik nachkommen, die die »produktive Offenheit « der Sinnsuche als performativen Prozess auffasst, mixed methods anwendet und nicht auf das fixierende Begreifen, sondern auf »Relationen des Erkennens « abhebt, ${ }^{19}$ eine Hermeneutik, die jene ästhetische Wende unterstützt, ebenso wie jene auf diese verweist.

\footnotetext{
Semir: Glanz und Elend des Gehirns. Neurobiologie im Spiegel von Kunst, Musik und Literatur. München: Reinhardt, 2010.

17 Grundlegend für die Ansätze des New Historicism bzw. Diskursanalyse: Greenblatt, Stephen: Shakespearean Negotiations: The Circulation of Social Energy in Renaissance England. Oxford: Clarendon Press, 1988.

$18 \mathrm{Zu}$ den Begriffen von Autologie bzw. Heterologie bzw. deren dynamische Relation vgl. das Forschungsprogramm des SFB 1391: https://uni-tuebingen.de/forschung/forschungsschwerpunkte/.

sonderforschungsbereiche/sfb-andere-aesthetik/forschungsprogramm/das-praxeologische-modell-einer-anderen-aesthetik/ (20.09.2021) bzw. Gerok-Reiter, Annette/Robert, Jörg: »Andere Ästhetik - Akte und Artefakte in der Vormoderne. Zum Forschungsprogramm des SFB 1391«. In: Gerok-Reiter u. a. (Hg.): Andere Ästhetik. Grundlagen - Fragen - Perspektiven. Berlin/Boston [erscheint 2022].

19 Bleumer u. a. (s. Anm. 4), S. 566 und 568.
} 
Beiden Ansätzen ist die Auffassung gemeinsam, dass Sinnsuche keineswegs den »Bändigungsversuch des Sinns $«{ }^{20}$ für das Verstehen impliziert, ${ }^{21}$ sondern umgekehrt die Forderung erhebt, eine »Bändigung« gerade nicht vorzunehmen. Darüber hinaus stellen beide ihren axiologischen Anspruch in neuer Weise aus: Eine Lektüre, die als gewinnbringend erachtet wird, und eine positive ästhetische Wertung spielen Hand in Hand. Und wie es nicht um eine »philosophische Hermeneutik post rem« geht, sondern um eine »literarische Hermeneutik in re ${ }^{22}$ so haben sich die Lektüren des Ästhetischen in ästhetische Lektüren zu verwandeln, nicht um in anachronistischer Weise sich >psychologisch $<$ fundierten Lesarten ${ }^{23}$ wieder zuzuwenden, sondern um »Texte als Ereignisse >anzunehmen «, indem mit Hilfe genauer literaturwissenschaftlicher Verfahrensweisen diese »in ihrer Bewirktheit und Einzigartigkeit zugleich rekonstruiert « werden können. ${ }^{24}$

»Hermeneutik heute « heißt somit zu realisieren, dass Verstehen dort einsetzt, wo in grundsätzlicher Weise der Einsicht Raum gegeben wird, dass jedes Verstehen im performativen Prozess des Lesens und Interpretierens nicht an eine Ende kommen kann - und dies aufgrund der nicht still zu stellenden Austauschverhältnisse und der Fülle an möglichen Austauschebenen gerade im historischen >Ereignis< des Textes selbst. ${ }^{25}$ Jedes Verstehen impliziert so das Eingeständnis eines Nicht-Verstehens, beruht auf einer Krise des Verstehens, ${ }^{26}$ ohne jedoch in einer »antihermeneutischen « grundsätzlichen Unlesbarkeit (Paul de Man) bzw. in einer »antihermeneutischen « grundsätzlichen Differenz, die kein Verstehen mehr erlaubt (Derrida), aufgehen zu müssen. ${ }^{27}$ Das Angebot von handhabbaren Lösungen, eindeutigen Intentionen oder fragloser Gewissheit - ein Angebot also, dem das Krisenmoment nicht eingeschrieben ist - macht heute im postfaktischen Zeitalter eher misstrauisch, nicht weil Lösungen nicht erwünscht wären, sondern weil gewusst wird, dass ein Verstehen, das kein Krisenmoment kennt, nicht ausreicht. Die Antwort hierauf kann jedoch weder sein,

20 Ebd., S. 569.

21 Zur Diskussion, anschließend an Blumenbergs >Bändigungsthese<, Gerok-Reiter, Annette: »Mythos und Ästhetik. Ordnungsgemengelagen des Erzählens in Veldekes >Eneasroman««. In: Daniela Fuhrmann/Pia Selmayr (Hg.): Erzählte Ordnungen - Ordnungen des Erzählens. Studien zu Texten vom Mittelalter bis zur Frühen Neuzeit. Berlin/Boston: de Gruyter, 2021, S. 275-304, hier S. 303 f. https://doi.org/10.1515/ 9783110729115.

22 Bleumer u. a. (s. Anm. 4), S. 579.

$23 \mathrm{Vgl}$. Schleiermacher (s. Anm. 3), S. $80 \mathrm{f}$.

24 Bogdal (s. Anm. 11), S. 156. Vgl. Bleumer, Hartmut: Ereignis. Eine narratologische Spurensuche im historischen Feld der Literatur. Würzburg: Königshausen \& Neumann, 2020.

25 Zu dieser Fülle vgl. auch Jung (s. Anm. 5), S. 177. Dies heißt jedoch - gegen Jung - keineswegs, dass es »keine richtige Deutung, bloß jeweils bessere Begründungen für einen Lektürevorschlag « geben könne (S. $177 \mathrm{f}$.). Auch das Angebot pluraler Lektürevorschläge bleibt auf die je adäquate, historisch kontextualisierte Deutung innerhalb des einen Vorschlags angewiesen; Pluralität sollte nicht mit Beliebigkeit verwechselt werden. Entsprechend betont Bogdal (s. Anm. 11), S. 155, denn auch gerade, dass die historische Kontextualisierung für eine »endliche Menge an Aussagemöglichkeiten« des Textes sorge. Ebendies kann und muss die konkrete Deutung anleiten.

26 Bleumer (s. Anm. 24), S. 25, bezeichnet dies als »Inkompossibilität«: Im »Ereignis tut sich vielmehr [...] ein Riss im Bereich des aktuell Möglichen auf, in dem schlagartig die unabsehbare Fülle aller weiteren erdenklichen Möglichkeiten spürbar wird.«

27 Rusterholz (s. Anm. 10), insbes. S. 167-176. 
das Bemühen um ein Verstehen ganz aufzugeben, noch Konfusion zum Normalzustand zu erklären. Als Antwort bietet sich vielmehr an, die Krise des Verstehens als Integral des Verstehens selbst aufzufassen. ${ }^{28}$

Genau dies aber gehört zum Kerngeschäft ästhetischen Verstehens. Im ästhetischen Verstehen ist die Krise des Verstehens gleichsam auf Dauer gestellt, nicht aber als Versagen, sondern als Konzept, um mehr verstehen zu können als das rational Handhabbare, das explizit Beabsichtigte, das begrifflich Fixierte. Dieses >mehr zielt heute nicht länger auf den exklusiven Raum derjenigen Kunst, die sich die Freiheit einer eigenen Gesetzlichkeit oder eines mit sich selbst identischen Textsinns nimmt. Es zielt vielmehr auf die Ambiguitäten, die entstehen, weil Semantiken nicht außerhalb der Pragmatik zu denken sind, weil ästhetische Lektüren nicht aus Lebenswelten hinaus-, sondern in sie hineinführen, weil Artefakte nicht allein autologisch, sondern mindestens ebenso heterologisch bestimmt sind. Eine >andere< Hermeneutik, eine Hermeneutik, die ihre Krise mitdenkt, ${ }^{29}$ kann so mit einer >anderen $\ll$ Ästhetik korrelieren, deren Verstehen nicht im Anspruch >interesselosen Wohlgefallens< oder reiner Präsenz aufgeht, ${ }^{30}$ sondern die das ästhetische Agieren und Verstehen als zentralen Erschließungsmodus im Rahmen komplexer historischer und sozialer Praktiken ${ }^{31}$ auffasst. ${ }^{32}$ Hermeneutische Lektüren des Ästhetischen werden so, im gelungenen Fall, zu ästhetischen Lektüren, ${ }^{33}$ die ästhetische Lektüren zu einer ästhetischen Praxis. ${ }^{34}$ Eine >andere< Hermeneutik hätte sich damit nicht nur den Status der Auslegungs-Kunst neu erobert, sondern hätte als Praxis einen gesellschaftlichen Ort allererst gefunden.

Funding Open Access funding enabled and organized by Projekt DEAL.

Open Access Dieser Artikel wird unter der Creative Commons Namensnennung 4.0 International Lizenz veröffentlicht, welche die Nutzung, Vervielfältigung, Bearbeitung, Verbreitung und Wiedergabe in jeglichem Medium und Format erlaubt, sofern Sie den/die ursprünglichen Autor(en) und die Quelle ord-

${ }^{28}$ Ebd., S. 176: »Die Alternative >Interpretierbarkeit oder Uninterpretierbarkeit der Texte ist wohl zu einfach.«

29 Hierin würde sich das Unternehmen der literarischen Hermeneutik, die sich nicht erst seit Jauß, sondern von ihren Anfängen an schrittweise immer »offener, pluraler« (Jung [s. Anm. 5], S. 176) zeigte, erst eigentlich ihrem Anspruch gerecht. In diesem Sinn hebt Bogdal (s. Anm. 11) hervor, dass manche "poststrukturalistische Globalkritik der Interpretation« der Hermeneutik, die nur auf die >eine< Wahrheit zulaufe, zu kurz greift (S. 138) - mit Verweis auf Blumenbergs ausgewogene Definition: »Hermeneutik geht auf das, was nicht nur je einen Sinn haben und preisgeben soll und für alle Zeiten behalten kann, sondern was gerade wegen seiner Vieldeutigkeit seine Auslegungen in seine Bedeutung aufnimmt «; Blumenberg, Hans: Die Lesbarkeit der Welt. Frankfurt a. M.: Suhrkamp, 1986, S. 21.

30 Dazu Gumbrecht (s. Anm. 7): »Letzten Endes wird in diesem Buch ein Verhältnis zu den Dingen dieser Welt befürwortet, das zwischen Präsenz- und Sinneffekten oszillieren könnte« (S. 12).

31 Vgl. Reckwitz, Andreas: Kreativität und soziale Praxis. Studien zur Sozial- und Gesellschaftstheorie. Bielefeld: transcript, 2016.

32 In diesem Sinn hat der SFB 1391 Andere Ästhetik ein praxeologisches Analysemodell entwickelt: s. Anm. 17.

33 Bleumer (s. Anm. 24), S. 7 f.: So könne auch Literaturwissenschaft »zum Ereignis werden. Gewiss heißt das nicht, dass ein solches wissenschaftliches Ereignis [...] dem literarischen gleichzusetzen wäre. Aber es beruht zumindest auf einer doppelten Grenzüberschreitung: von der Literatur zur Wissenschaft und von der Wissenschaft zur Literatur.«

34 Vgl. Bertram, Georg W.: Kunst als menschliche Praxis. Eine Ästhetik. Berlin: Suhrkamp, 2. Aufl. 2018. 
nungsgemäß nennen, einen Link zur Creative Commons Lizenz beifügen und angeben, ob Änderungen vorgenommen wurden.

Die in diesem Artikel enthaltenen Bilder und sonstiges Drittmaterial unterliegen ebenfalls der genannten Creative Commons Lizenz, sofern sich aus der Abbildungslegende nichts anderes ergibt. Sofern das betreffende Material nicht unter der genannten Creative Commons Lizenz steht und die betreffende Handlung nicht nach gesetzlichen Vorschriften erlaubt ist, ist für die oben aufgeführten Weiterverwendungen des Materials die Einwilligung des jeweiligen Rechteinhabers einzuholen.

Weitere Details zur Lizenz entnehmen Sie bitte der Lizenzinformation auf http://creativecommons.org/ licenses/by/4.0/deed.de. 